\title{
Recherche-action en contexte de projet plein air interdisciplinaire au primaire: nature des savoirs développés par les praticiens et réflexions méthodologiques
}

Par Andrée Lessard, Ph. D., Université du Québec à Chicoutimi

\section{Résumé}

Alors que la recherche participative est considérée comme un vecteur de développement professionnel chez les enseignants (Couture, Bednarz et Barry, 2007; Guillemette et Savoie-Zajc, 2012), cet article a pour but d'illustrer la nature des savoirs mobilisés par les praticienschercheurs qui se sont engagés dans une recherche-action. Dans un contexte de projet plein air interdisciplinaire, les enseignants et la directrice d'une petite école en région éloignée au Saguenay ont cherché à augmenter le niveau de motivation en lecture des garçons. Par l'intermédiaire de la recherche-action, elles ont dégagé des pistes d'intervention qu'elles ont pu intégrer à leur enseignement, contribuant ainsi à mobiliser des savoirs d'une nature multidimensionnelle, d'origines différentes et qui touchent autant la théorie que la pratique. Les résultats seront discutés, puis suivis d'une réflexion en lien avec le choix méthodologique de la recherche-action et d'un survol de ses retombées.

Mots-clés: Recherche-action en éducation, développement des compétences professionnelles, formation continue par la recherche, projet interdisciplinaire au primaire, plein air à l'école, motivation des garçons en lecture, nature des connaissances développées par la recherche-action 


\section{REVUE HYBRIDE DE L'ÉDUCATION}

\section{Introduction}

Dans le cadre de la recherche en sciences de l'éducation, la recherche participative est considérée comme un vecteur de développement professionnel (Couture, Bednarz et Barry, 2007; Guillemette et Savoie-Zajc, 2012). Cet article a pour but d'interroger la nature des savoirs développés par les praticiens-chercheurs dans un projet de recherche-action dont l'objectif était d'améliorer la motivation en lecture de jeunes garçons du primaire. La problématique, le cadre conceptuel, la méthodologie et les résultats de ce projet seront présentés. Les savoirs mobilisés par les praticiens chercheurs seront ensuite décrits. Pour conclure, des pistes de réflexion quant à l'option méthodologique de la recherche-action seront proposées.

\section{Problématique}

La recherche-action présentée dans cet article tire son origine d'une demande provenant des praticiennes d'une petite école primaire (environ 45 élèves répartis en 4 classes : maternelle, $1^{\mathrm{er}}, 2^{\mathrm{e}}$ et $3^{\mathrm{e}}$ cycles) située en région éloignée sur le territoire du Saguenay-Lac-St-Jean dans laquelle un projet interdisciplinaire plein air a été implanté. Cette demande faisait état de différents objectifs :

1. Favoriser la motivation scolaire et l'estime de soi des élèves;

2. Contribuer à l'attrait de nouveaux élèves ou à la rétention de la clientèle à l'école du village en valorisant un projet de plein air auquel chaque classe participait (une demi-journée par semaine consacrée à des activités comme le vélo, l'hébertisme, le ski, la raquette, l'orientation en forêt, les techniques de survie, etc.) les préparant à une grande sortie à la fin de l'année scolaire pour le $3^{e}$ cycle (expédition de trois jours avec camping).

Le projet était propice à l'intégration de plusieurs matières, dont la lecture, les mathématiques, les sciences et l'éducation physique (par exemple, lire un texte sur la thermorégulation permettait aux élèves de 


\section{$\&$}

\section{REVUE HYBRIDE DE L'ÉDUCATION}

bien choisir leurs vêtements au moment de sortir à l'extérieur, ce qui touchait à la fois la lecture et les sciences). Selon la directrice, les garçons semblaient moins motivés en lecture et leurs résultats scolaires étaient moins élevés, ce qui a servi de point de départ à l'intention de l'équipe-école de documenter les effets de leurs pratiques à l'intérieur du projet interdisciplinaire plein air sur la motivation à lire des élèves masculins.

La motivation est une composante essentielle de la réussite scolaire, entre autres dans la discipline du français, langue d'enseignement (Bouffard, Brodeur et Vezeau, 2005). En effet, la perception qu'ont les élèves de leur efficacité en lecture, du plaisir de lire, de l'importance accordée à la lecture, du lien entre la lecture et les notes scolaires et de la reconnaissance obtenue par la lecture est significativement corrélée à la réussite en lecture (Logan et Medford, 2011; Mucherah et Yoder, 2008). Or, chez les garçons du primaire, il semblerait que la motivation intrinsèque en lecture, la valeur qu'ils accordent à l'acte de lire et la motivation à satisfaire les attentes d'autrui et à obtenir de bonnes notes en lecture s'avèrent toutes moins élevées que chez les filles (Bouffard et al., 2005; McGeown, Goodwin, Henderson et Wright, 2012; Mucherah et Yoder, 2008; Senn, 2012). Pour remédier à la situation, plusieurs approches d'enseignement visant à développer la motivation extrinsèque en lecture par l'intermédiaire de moyens diversifiés ont été mises en place, favorisant les récompenses pour chaque lecture accomplie (Everhart, 2005). Toutefois, les recherches indiquent que ce genre de programme associe la lecture à l'évaluation, ne permettant pas nécessairement le développement de la motivation intrinsèque et du plaisir à lire (Serafini, 2013). Pour changer cette dynamique, il est possible de penser, entre autres, aux approches par projet, qui rehaussent la motivation scolaire (Proulx, 2004). De plus, les recherches indiquent qu'une approche interdisciplinaire incluant l'éducation physique a permis d'accroitre la motivation en lecture d'élèves masculins (Sharp et al., 2003). Par ailleurs, les effets de programmes d'éducation en plein air 


\section{$\&$}

\section{REVUE HYBRIDE DE L'ÉDUCATION}

ont été documentés et ont permis d'identifier des retombées positives sur le sentiment d'auto-efficacité et la perception de soi (Gargano, 2010). Nous avons donc conjointement décidé de nous intéresser au développement de la dynamique motivationnelle en lecture des garçons du primaire dans un contexte précis de projet interdisciplinaire en plein air, car il était possible de croire que les élèves allaient gagner en motivation dans ce contexte.

\section{Cadre conceptuel}

Pour répondre aux objectifs ciblés, les praticiennes ont dû s'approprier ou approfondir plusieurs concepts, dont deux qui ont soutenu toute la démarche de recherche-action : la dynamique motivationnelle en lecture et l'interdisciplinarité.

Premièrement, la dynamique motivationnelle fait référence à un concept qui évolue dans le temps en fonction de la situation et du contexte (Bouffard et al., 2005; Guay, Chanal, Ratelle, Marsh, Larose et Boivin, 2010; Ryan et Deci, 2000, 2002; Viau, 2009). Elle est décrite comme un «phénomène qui tire sa source dans des perceptions que l'élève a de lui-même et de son environnement, et qui a pour conséquence qu'il choisit de s'engager à accomplir l'activité pédagogique qu'on lui propose et de persévérer dans son accomplissement, et ce, dans le but d'apprendre » (Viau, 2009, p. 12). Selon cette description, pour mieux comprendre ce qui motive les garçons à s'engager dans des activités de lecture, il importe d'évaluer leurs perceptions de la valeur de ces activités (intérêt et utilité), de leur compétence à les accomplir et de leur contrôlabilité sur les tâches demandées. La caractéristique de mouvement et d'évolution permet de distinguer la dynamique motivationnelle de la motivation en soi. Elle permet de mettre en évidence que la motivation en lecture peut varier en intensité et en qualité dans le temps. Selon la théorie de l'autodétermination (Ryan et Deci, 2000/2002), la motivation constitue un ensemble de raisons qui nous poussent à adopter certains comportements. II existe deux principaux types de 


\section{REVUE HYBRIDE DE L'ÉDUCATION}

motivation : intrinsèque (plaisir et satisfaction rattachés à l'activité) et extrinsèque (raisons instrumentales de s'engager dans l'activité). Cette dernière peut se faire par régulation externe (récompense ou reconnaissance), ou encore par régulation identifiée, soit lorsque l'objectif est inhérent à l'apprenant, mais qu'il découle de pressions internes extérieures au plaisir de lire (par exemple, pour apprendre, pour pouvoir lire à ses enfants plus tard, pour éviter de se sentir coupable, etc.).

Deuxièmement, l'interdisciplinarité serait une pratique éducative qui fait appel à plus d'une perspective disciplinaire, dont les relations entre disciplines peuvent s'effectuer sur le plan didactique (Larose et Lenoir, 1998; Lowe, 2002). Ces relations peuvent s'effectuer par des liens de complémentarité en ayant comme point commun soit un objet d'étude, des concepts, des notions ou des démarches d'apprentissage, pour ne mentionner que ces exemples (Larose et Lenoir, 1998). Dans le cadre du projet plein air (thème commun avec notions s'y rattachant), les disciplines incluses sont principalement les sciences, les mathématiques, le français (lecture) et l'éducation physique.

Ce travail conceptuel nous a permis de définir nos objectifs opérationnels :

1. Décrire la dynamique motivationnelle des garçons en lien avec la lecture pendant leur participation au projet plein air;

2. Dégager des pistes pertinentes d'intervention en lien avec la dynamique motivationnelle des garçons;

3. Intégrer les pistes d'intervention soulevées à l'intérieur du projet interdisciplinaire pendant l'année scolaire 2014-2015.

La dynamique motivationnelle a été décrite à deux moments, soit au début de l'implantation du projet plein air (automne 2012) et après presque deux ans de participation à ce projet (hiver 2014). Ces portraits ont permis aux praticiennes de participer activement à l'atteinte des objectifs 2 et 3 , sur lesquels nous allons nous centrer pour mettre en 


\section{8}

\section{REVUE HYBRIDE DE L'ÉDUCATION}

évidence la nature des savoirs qu'elles ont mobilisés à l'intérieur de la recherche-action.

\section{Méthodologie}

Parmi les modèles de recherche participative, c'est une recherche-action qui a été mise en œuvre (Anadón et Savoie-Zajc, 2007; Couture et al., 2007; Groulx, 1997; Guillemette et Savoie-Zajc, 2012; Stoloff et Beaudoin, 2012). En effet, un pôle d'action et un pôle de recherche ont été articulés dans une démarche ordonnée et systématique caractérisée par l'alternance entre la pratique et la réflexion, menant ainsi à une transformation des pratiques (Stoloff et Beaudoin, 2012). Selon Dolbec et Clément (2004), elle répond aux trois principaux critères d'une recherche-action: autant la chercheuse que les professionnelles ont participé activement à la recherche de solutions et de pistes d'intervention afin de motiver davantage les garçons en lecture, ce sont les professionnelles elles-mêmes qui ont mis en place ces changements dans leur propre pratique et des mesures ont été prises afin d'assurer la pérennité de ces changements. Comme les données recueillies sont principalement de nature qualitative, mais également quantitative (questionnaire), une méthodologie mixte a été employée.

\section{Participants}

Les acteurs impliqués dans la recherche-action sont les quatre enseignantes titulaires, l'enseignante spécialiste d'éducation physique et la directrice de l'école. En complément, tous les garçons de l'école $(n=$ 15) ont participé pour dresser un portrait de leurs profils de dynamique motivationnelle en lecture. De plus, un conseiller pédagogique a participé à l'une des phases de la recherche.

\section{Instrumentation}

Un questionnaire écrit (Guay et al., 2010) et des entretiens individuels semi-dirigés (Poupart, 1997), complétés par des observations en classe et en plein air, ont d'abord permis de décrire la dynamique 


\section{REVUE HYBRIDE DE L'ÉDUCATION}

motivationnelle des garçons en lien avec la lecture. Le portrait ainsi obtenu a servi à répondre au premier objectif du projet. Pour dégager des pistes d'intervention pertinentes, ce qui correspondait au deuxième objectif, les enseignantes ont été invitées à remplir un journal de bord au fur et à mesure qu'elles vivaient la mise en place du projet interdisciplinaire avec leurs élèves, sans contrainte de fréquence de rédaction. Des pistes de réflexion leur étaient fournies (par exemple, notez les activités qui ont bien fonctionné et expliquez pourquoi, notez vos observations en lien avec la motivation en lecture des garçons, etc.). De plus, des questions individuelles leur ont été adressées afin de poursuivre leur réflexion et de préparer le groupe de discussion (Geoffrion, 2003) qui a eu lieu à l'automne 2014 et auquel un conseiller pédagogique s'était joint. Pour atteindre le troisième objectif, soit intégrer les pistes d'intervention retenues à l'intérieur du projet interdisciplinaire, des groupes de discussion et des entretiens individuels semi-dirigés (Geoffrion, 2003; Poupart, 1997) ont été utilisés à plusieurs reprises pendant l'année scolaire 2014-2015. C'est pendant cette période qu'une démarche de co-construction des connaissances et de transformation des pratiques a été implantée.

\section{Analyse des résultats}

Les données recueillies auprès des élèves par questionnaire ont fait l'objet de statistiques descriptives. Les entretiens ont été transcrits et ont fait l'objet d'une analyse de contenu (L'écuyer, 1990). Pour chaque élève, les données recueillies par questionnaire, par entretien et par observation ont été croisées afin d'obtenir un portrait des profils de dynamique motivationnelle en lecture des garçons. C'est ce portrait qui a été présenté à l'équipe-école afin de stimuler les échanges pour l'atteinte des objectifs 2 et 3 .

Pour le deuxième et le troisième objectifs, les informations qui devaient être contenues par écrit dans le journal de bord ont été récoltées verbalement lors du groupe de discussion à l'automne 2014. Elles ont été 


\section{REVUE HYBRIDE DE L'ÉDUCATION}

mises en relation avec l'ensemble des résultats recueillis jusqu'à cette date afin de mieux documenter la situation et de permettre une certaine triangulation de la collecte des données (Cohen, Manion et Morrison, 2000). Tous les groupes de discussion et tous les entretiens individuels ont été enregistrés et transcrits. Ils ont fait l'objet d'une démarche d'analyse de contenu (L'écuyer, 1990) en employant une thématisation en continue (Paillé et Mucchielli, 2012), ce qui a permis de faire émerger des pistes d'intervention (objectif 2) et de les intégrer au projet interdisciplinaire (objectif 3). C'est l'analyse de ces données qui va permettre, dans cet article, de documenter le processus de transformation des pratiques.

\section{Résultats et discussion}

Premier objectif : décrire la dynamique motivationnelle des garçons en lien avec la lecture pendant leur participation au projet plein air

Nous n'allons pas donner une description détaillée de ces résultats dans cet article. Néanmoins, il a été mis de l'avant que le sentiment de compétence en lecture est variable (par exemple, " y’a rien de difficile, sauf quelques mots des fois / le français, c'est pas comprenable »), que la motivation intrinsèque en lecture demeurait la dimension la moins développée, et ce, aux différents moments de collecte (par exemple, "J'aime pas la lecture, j'trouve ça plate ") et que, même si les garçons reconnaissent généralement que savoir lire est important (par exemple, «La lecture c'est important »), l'utilité de savoir lire était très difficile à préciser (par exemple, “c'est important pour savoir lire ») ou constituait un but très éloigné (par exemple, « plus tard, j'vais pouvoir lire des histoires à mes enfants mettons »).

Deuxième objectif : formuler des pistes pertinentes d'intervention

À la suite du portrait qui leur a été présenté sur la dynamique motivationnelle des garçons, les enseignantes, la directrice et le conseiller pédagogique ont soulevé plusieurs pistes d'intervention afin de mieux 


\section{REVUE HYBRIDE DE L'ÉDUCATION}

répondre aux besoins de leurs élèves. Des pistes plus générales ont d'abord été soulevées en lien avec la pérennité du projet plein air une fois la recherche terminée, dont la création de planifications réutilisables sur deux ans (classes-cycles), l'intégration des contenus des programmes disciplinaires et du cadre théorique de la recherche dans la planification et la prise en compte de mesures de sécurité.

Des pistes ont ensuite été formulées plus spécifiquement en réponse à la dynamique motivationnelle en lecture des garçons. À cet égard, les pistes qui ont été priorisées par les praticiennes sont :

1. Utiliser davantage la lecture afin de préparer les sorties plein air en choisissant des thèmes de lecture y étant étroitement reliés;

2. Expliciter les liens entre différents formats de lecture et leur utilité à court terme (rallye, affiches, Internet, expériences, etc.);

3. Utiliser des textes incitatifs et interactifs;

4. Reconnaitre les réussites des élèves en lecture et féliciter l'effort;

5. Faire la lecture aux élèves;

6. Proposer des textes adaptés au niveau des élèves qui ne sont pas trop longs.

Ces pistes ont servi d'assise au changement des pratiques faisant l'objet du troisième objectif de la recherche.

Troisième objectif : Intégrer les pistes d'intervention soulevées à l'intérieur du projet interdisciplinaire

La mise en application des pistes soulevées précédemment s'est faite par un processus itératif selon lequel l'équipe de recherche (chercheuse et assistants) et l'équipe-école (praticiennes) se sont rencontrées plusieurs fois. Chacune devait accomplir des tâches précises à tour de rôle ou conjointement. La collaboration s'est donc avérée essentielle afin d'accompagner le changement des pratiques désiré et de 


\section{REVUE HYBRIDE DE L'ÉDUCATION}

permettre une appropriation de la démarche de recherche par les enseignantes.

Comme point de départ, l'équipe de recherche a proposé deux canevas pour faciliter la planification du projet interdisciplinaire: le premier pour des séquences didactiques (grandes lignes d'une planification s'étalant de deux à quatre leçons, selon le thème), le deuxième pour chacune des leçons détaillées. Ils fournissaient l'espace pour prévoir les liens avec les programmes disciplinaires. Pendant une séance de travail d'équipe, les enseignantes ont proposé des modifications à ces canevas afin de mieux répondre à leurs besoins. Une fois les canevas définis, la chercheuse a rencontré individuellement chaque titulaire afin de co-construire avec elle une séquence générale de leçons portant sur la thermorégulation. Ces séances ont fourni un plan de travail pour l'équipe de recherche, qui s'est chargée de détailler les contenus des planifications sur le thème de la thermorégulation en $y$ incluant les éléments du cadre conceptuel de la recherche et les liens théoriques soulevés par les pistes retenues. Chaque enseignante devait ensuite modifier les planifications pour mieux répondre à ses besoins pratiques, fournissant du même coup des commentaires dont l'équipe de recherche a tenu compte pour les planifications subséquentes. Le processus s'est répété, séquence par séquence, pour les thèmes retenus (orientation, alimentation, papillons, etc.). Les enseignantes devaient par la suite faire vivre les activités à leurs élèves, leur permettant ainsi d'apporter d'autres modifications aux planifications, au besoin, afin de les réutiliser deux ans plus tard.

Transformation des pratiques: changements observés dans les planifications

Le travail réalisé pour atteindre les trois objectifs permet de documenter les transformations de pratiques auxquelles le projet a donné lieu. Une analyse sommaire des planifications réalisées avant et après le processus itératif mis en place à l'intérieur de la recherche-action a révélé 


\section{8}

\section{REVUE HYBRIDE DE L'ÉDUCATION}

qu'au départ, soit avant l'automne 2014, les planifications ne contenaient pas toujours de liens explicites précis avec les programmes disciplinaires. Les activités étaient parfois liées aux intérêts spontanés des élèves, elles visaient à motiver les élèves « en général » et elles étaient pilotées selon l'expérience des enseignantes (de façon « intuitive ", pour reprendre leurs termes). À l'hiver 2015, soit après le début du processus itératif décrit plus haut, les planifications analysées contenaient des liens étroits avec les contenus disciplinaires dans une structure interdisciplinaire (chaque leçon incluait des contenus d'au moins deux disciplines). Cette caractéristique permet d'envisager un plus petit investissement de temps au moment de réutiliser les planifications au bout de deux ans, mais également d'assurer la validité des contenus présentés en lien avec le Programme de formation de l'école québécoise et la Progression des apprentissages selon le niveau (MELS, 2009), d'une part, et d'autre part, en lien avec les connaissances issues des recherches en didactique (Simard, Dufrays, Dolz et Garcia-Debanc, 2010). De plus, les planifications permettaient une adaptation aux intérêts et aux réactions des élèves, mais l'enseignement n'était pas improvisé. Chaque action était justifiée en fonction du cadre conceptuel de la recherche ou du profil de dynamique motivationnelle des garçons, tenant compte ainsi des résultats obtenus au premier objectif. À titre d'exemple, pour favoriser la prise de conscience de l'utilité de la lecture au quotidien, il était planifié d'indiquer aux élèves, avant une lecture, que ce qu'ils liraient leur permettrait d'apprendre sur les éléments nutritifs contenus dans les aliments. Pour faciliter le transfert des connaissances, il était planifié de choisir adéquatement une bonne collation lors d'une sortie plein air. Mettre en évidence l'utilité de la lecture vient renforcer la motivation à s'engager dans la tâche (Viau, 2009), liant ainsi le cadre conceptuel de la recherche aux profils motivationnels des garçons de l'école. 


\section{REVUE HYBRIDE DE L'ÉDUCATION}

\section{Nature des savoirs développés et déclarés par les praticiens du milieu scolaire}

Un questionnaire écrit (rempli collectivement pendant un groupe de discussion) a porté sur les savoirs développés par les enseignantes et la directrice, plus précisément sur les apprentissages qu'elles déclarent avoir réalisés en participant activement à la recherche. Selon l'analyse de contenu par thématisation en continue, ils ont été regroupés en cinq dimensions :

1. Savoirs liés à la dynamique motivationnelle en lecture des garçons qui découlent des résultats de la recherche (premier objectif);

2. Savoirs liés à la dynamique motivationnelle en lecture des garçons qui découlent d'observations directes;

3. Savoirs liés aux pratiques pouvant influencer la dynamique motivationnelle en lecture;

4. Savoirs liés à la théorie sur la dynamique motivationnelle en lecture et l'interdisciplinarité;

5. Savoirs liés à la démarche de recherche-action en éducation.

Pour la première dimension, le portrait présenté à l'équipe-école a permis d'en apprendre davantage sur les différentes facettes de la dynamique motivationnelle en lecture de leurs élèves garçons. Entre autres, les enseignantes ont pu constater que les garçons ont obtenu des résultats légèrement plus élevés, mais non de façon significative, à l'hiver 2014 qu'à l'automne 2012 pour toutes les dimensions de la dynamique motivationnelle en lecture qui ont été mesurées : l'intensité du niveau de motivation semblait s'être au moins maintenue dans le temps, allant ainsi à l'encontre de la littérature scientifique selon laquelle la force de la motivation en lecture tend à décroitre avec l'avancée des élèves dans leur cheminement primaire (Giasson, 2011). Les nuances apportées par les entretiens individuels indiquaient aux praticiennes que les élèves 


\section{REVUE HYBRIDE DE L'ÉDUCATION}

masculins reconnaissaient l'importance de la lecture dans la vie en général, mais qu'ils n'étaient pas en mesure d'identifier pourquoi en faisant des parallèles avec leur quotidien (par exemple, "ils ne sont pas capables de dire à quoi ça sert de savoir lire »). Elles ont soulevé aussi qu'ils ne semblaient pas réaliser la présence de tâches de lecture dans le cadre du projet plein air, alors que pourtant ils lisaient à des fins utiles (par exemple, " ils lisent, mais parce qu'ils sont dehors en train de faire le rallye, ils s'en rendent pas compte»). Finalement, les praticiennes ont retenu que les garçons préféraient les textes plus courts et que parfois, ils lisaient davantage en lien avec des thèmes qui les intéressaient même si la lecture en soi ne suscitait pas beaucoup d'intérêt (par exemple, «ils aimaient vraiment lire sur les champignons, parce que ça venait d'eux : il $y$ en avait beaucoup et on est parti avec cette thématique-là pour suivre l'intérêt des élèves »).

Pour la deuxième dimension, les enseignantes affirment que leur participation à la recherche les a amenées à observer plus attentivement leurs élèves masculins afin d'en dégager des constats pouvant appuyer les pistes d'intervention à proposer par la suite. Ces observations ont donc contribué à développer une meilleure connaissance des élèves en complétant le portrait dressé initialement. Par exemple, elles ont identifié que les garçons semblaient plus motivés à lire en contexte d'interaction ou en action et qu'ils appréciaient le côté ludique des activités plein air (par exemple, "ils aiment vraiment faire le plein air. Ils savent qu'ils sont là pour apprendre, mais ils ne se rendent pas compte qu'ils travaillent [la lecture] pendant qu'ils sont dehors "). Elles ont soulevé également que l'apport de nouvelles connaissances et le sujet des lectures proposées s'avéraient déterminants pour la motivation (par exemple, «ils aimaient vraiment apprendre sur les champignons»). Finalement, elles ont remarqué une plus grande implication des élèves en lecture lorsqu'une personne-ressource invitée les rencontrait pour leur apporter de nouvelles connaissances (par exemple, «le biologiste qui faisait gouter des plantes comestibles »). 


\section{REVUE HYBRIDE DE L'ÉDUCATION}

Pour la troisième dimension, les pratiques pouvant influencer la dynamique motivationnelle en lecture ont fait l'objet de nombreuses discussions et de mises à l'essai. Les réflexions collectives ou individuelles ont été mises en commun et elles ont orienté les choix d'intervention à prioriser, à mettre en œuvre ou à modifier dans l'enseignement. En combinant à la fois les connaissances pratiques documentées par la recension des écrits scientifiques, l'expérience des enseignantes, leurs observations et les données recueillies directement auprès des garçons (questionnaires, entretiens), les participantes ont été en mesure d'identifier de nouveaux savoirs liés à la pratique. Ainsi, pour appuyer la transformation de leurs pratiques dans l'optique d'influencer la dynamique motivationnelle en lecture chez les garçons, elles ont choisi de proposer des lectures sur des sujets qui les intéressaient particulièrement, par exemples, les champignons, tout en tentant de les garder en action, comme pour la lecture d'une expérience à réaliser (par exemple, «on devrait favoriser les textes incitatifs »). Elles ont également choisi de nommer explicitement l'intention de lecture avant d'entreprendre une activité dans le but d'aider les garçons à prendre conscience que la lecture est omniprésente et très utile dans de nombreux domaines, y compris en plein air (par exemple, "on pourrait leur dire que s'ils comprennent bien les consignes [écrites], ils vont réussir l'activité, ils vont réussir le rallye. / On va leur poser la question: quand tu utilises la tablette, est-ce que c'est de la lecture ? »). Dans cette lignée, elles ont également choisi de leur donner des exemples concrets de l'usage qu'ils peuvent faire à court terme des apprentissages qu'ils sont en train de réaliser. Finalement, elles ont mentionné que sur le plan pratique, le développement des planifications détaillées permettait la continuité du projet plein air en constituant un recueil de notions et d'activités réutilisables (par exemple, "on va pouvoir les reprendre dans deux ans sans avoir à tout recommencer »).

Pour la quatrième dimension, les enseignantes ont identifié que participer à l'élaboration et à la modification de planifications tenant 


\section{REVUE HYBRIDE DE L'ÉDUCATION}

compte d'un cadre théorique sur la dynamique motivationnelle en lecture et l'interdisciplinarité leur a permis de « renouer avec les assises des programmes disciplinaires" tout en favorisant une gradation des apprentissages entre les cycles. Elles ont également spécifié que les justifications théoriques venaient les conforter dans plusieurs de leurs pratiques actuelles et qu'elles ajoutaient de la valeur à leur travail : elles étaient alors en mesure d'expliquer pourquoi le choix de leurs pratiques était adéquat. Entre autres, elles ont mentionné avoir précisé les concepts liés à la perception de la valeur et de l'utilité d'une tâche de lecture (motivation) et aux liens pouvant exister entre plusieurs disciplines dans l'atteinte des objectifs poursuivis dans le projet plein air (interdisciplinarité).

Pour la cinquième dimension, les praticiennes ont acquis des savoirs sur la recherche-action en éducation. D'abord, elles ont reconnu avoir contribué à l'avancement des connaissances de nombreuses façons, entre autres en participant aux discussions, en suggérant des pistes d'intervention à la suite des premiers résultats obtenus, en faisant part de leurs observations et en mettant en pratique les pistes suggérées pour influencer la dynamique motivationnelle en lecture des garçons. Elles ont également réalisé que la recherche-action permettait tant à l'équipe-école qu'à l'équipe de recherche de se fixer des objectifs communs et de diriger les efforts dans une même direction. La recherche a également permis aux enseignantes de prendre connaissance des résultats positifs associés au travail qu'elles accomplissaient déjà, ce qui a eu pour effet de les stimuler et de les motiver davantage à poursuivre leur participation active à la recherche (par exemple, "c'est beaucoup de travail, [...] mais le fait de voir les résultats positifs de notre travail, ça nous motive à poursuivre dans la même direction, c'est valorisant »).

En réponse au but poursuivi par cet article, il est possible d'avancer que les savoirs mobilisés par les praticiens du milieu scolaire 


\section{REVUE HYBRIDE DE L'ÉDUCATION}

sont d'une nature multidimensionnelle, qu'ils ont des origines différentes et qu'ils touchent autant la théorie que la pratique.

\section{Conclusion}

Les changements de pratique pour influencer la dynamique motivationnelle en lecture des garçons dans un contexte de projet plein air ont été le fruit d'une participation active de plusieurs personnes. Par un processus itératif qui impliquait des discussions, des réflexions, des échanges et des tâches partagées entre l'équipe de recherche et l'équipeécole, des planifications détaillées ont été créées dans l'atteinte de deux principaux buts communs :

1. Influencer la dynamique motivationnelle en lecture des garçons;

2. Valoriser le projet de plein air.

Cette co-construction des savoirs, dont la nature est multidimensionnelle, s'est faite à l'intérieur des options méthodologiques qui ont été retenues en fonction du déroulement de la recherche. Parmi celles-ci, l'option du modèle de recherche participative retenu, soit la recherche-action, a fait l'objet d'une réflexion au moment où ce choix a été arrêté. C'est seulement après les premières rencontres entre chercheur et praticiens que la formulation du troisième objectif, qui impliquait « d'intégrer des pistes à son enseignement », qu'un volet action a pu se dessiner à la recherche. Par contre, pour confirmer le choix du format recherche-action, il a fallu que le processus itératif décrit plus haut s'instaure, assurant ainsi une structure permettant l'alternance entre l'action et la pratique réflexive (Stoloff et Beaudoin, 2012). Ce format n'aurait pas pu être retenu si, par exemple, les praticiennes avaient choisi d'appliquer en classe des planifications fournies par l'équipe de recherche sans s'arrêter pour réfléchir, pour observer, pour repenser, pour modifier ou redéfinir de nouvelles planifications visant à motiver leurs élèves masculins en lecture. Les trois principaux critères suggérés par Dolbec et Clément (2004) pour définir une recherche-action ont été remplis, mais 


\section{REVUE HYBRIDE DE L'ÉDUCATION}

leur forme précise s'est dessinée pendant le déroulement de la recherche. Au début, sans savoir quelle était la direction - au moins générale - qui serait prise par la transformation des pratiques, il semblait impossible de choisir déjà les moyens assurant la pérennité de ces changements. Des planifications sur deux ans avec un contenu très structuré et appuyé par la recherche ont constitué un outil facile à réutiliser permettant l'atteinte de ce critère.

La recherche-action détient une grande part d'imprévisibilité découlant entre autres de la complexité et de l'évolution des réflexions individuelles et collectives des praticiens pour mener à de nouveaux savoirs (Stoloff et Beaudoin, 2012). Les allers-retours entre travail individuel et travail collectif, entre réflexions et actions, entre questions et analyses de situations ont permis d'en arriver à retenir des solutions qui semblent influencer la dynamique motivationnelle en lecture des élèves, suscitant ainsi le désir de remettre en application les connaissances acquises ou développées dans le futur. C'est pourquoi il a semblé opportun, dans ce cas-ci, de laisser du temps au processus de recherche participative avant d'arrêter le choix du format sur une recherche-action : il fallait s'assurer d'abord du désir de pérennité des changements. La flexibilité nécessaire pour mener ce type de recherche s'est donc illustrée par l'attente d'un regroupement de critères spécifiques, définis en fonction de l'évolution de la recherche et de son caractère participatif, avant d'opter pour une structure et une organisation pouvant soutenir une recherche-action.

Malgré les défis qui l'accompagnent, la recherche-action entraine des retombées sur les plans pratique et scientifique. Tels que déclarés par les praticiennes, les savoirs liés à leur participation à la recherche sont nombreux et touchent à plusieurs dimensions, contribuant ainsi à leur développement professionnel. Par contre, le fait qu'ils soient déclarés limite la généralisation des dimensions soulevées à d'autres recherchesactions. Les retombées se situent aussi sur le plan scientifique par le 


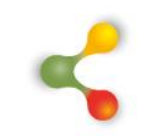

\section{REVUE HYBRIDE DE L'ÉDUCATION}

développement de connaissances ayant émergé dans un contexte éducatif particulier de projet interdisciplinaire en plein air, ouvrant ainsi la porte à des réflexions nouvelles portant sur les conditions pouvant favoriser la motivation en lecture des garçons. Finalement, la participation de la chercheuse au processus de transformation des pratiques afin de résoudre un problème ou d'atteindre un but commun contribue également à raffermir les liens qui unissent la pratique et la recherche, ancrant ainsi les connaissances développées dans un contexte scolaire réel. 


\section{REVUE HYBRIDE DE L'ÉDUCATION}

\section{Références}

Anadón, M. et Savoie-Zajc, L. (2007). La recherche-action dans certains pays anglo-saxons et latino-américains. Une forme de recherche participative. Dans M. Anadón (dir.), La recherche participative ( $\mathrm{p}$. 11-30). Québec, QC : PUQ.

Bouffard, T., Brodeur, M. et Vezeau, C. (2005). Les stratégies de motivation des enseignants et leurs relations avec le profil motivationnel d'élèves du primaire. Rapport de recherche. Repéré à file:///C:/Users/a1lessar/AppData/Local/Microsoft/Windows /INetCache/IE/ZX4XFEQK/Les strategies de motivation des enseignants et le.pdf

Cohen, L., Manion, L. et Morrison, K. (2000). Research methods in Education (5e éd.). New York, NY : Routledge/Falmer.

Couture, C., Bednarz, N. et Barry, S. (2007). Multiples regards sur la recherche participative. Une lecture transversale. Dans M. Anadón (dir.), La recherche participative (p. 205-221). Québec, QC : PUQ.

Dolbec, A. et Clément, J. (2004). La recherche-action. Dans T. Karsenti et L. Savoie-Zajc (dir.), La recherche en éducation: étapes et approches (p. 181-208). Sherbrooke, QC : Éditions du CRP.

Everhart, N. (2005). A crosscultural inquiry into the levels of implementation of accelerated reader and its effect on motivation and extent of reading: perspectives from Scotland and England. Chicago, IL : American Library Association.

Gargano, V. (2010). Les retombées du cours collégial "Plein air expérentiel » aux plans personnel et interpersonnel (Mémoire de maitrise inédit). Université du Québec à Chicoutimi.

Geoffrion, P. (2003). Le groupe de discussion. Dans B. Gauthier (dir.), Recherche sociale. De la problématique à la collecte des données (p. 333-356). Québec, QC : Presses de I'Université du Québec. 


\section{REVUE HYBRIDE DE L'ÉDUCATION}

Giasson, J. (2011). La lecture. Apprentissage et difficultés. Montréal, QC : Gaëtan Morin.

Groulx, L.-H. (1997). Contribution de la recherche qualitative à la recherche sociale. Dans J. Poupart (dir.), La recherche qualitative. Enjeux épistémologiques et méthodologiques (p. 55-84). Montréal, QC : Gaëtan Morin.

Guay, F., Chanal, J., Ratelle, C., Marsh, H. W., Larose, S. et Boivin, M. (2010). Intrinsic, identified, and controlled types of motivation for school subjects in young elementary school children. Bristish Journal of Educational Psychology, 80, 711-735.

Guillemette, S. et Savoie-Zajc, L. (2012). La recherche-action et ses rapports de coconstruction de savoirs et de formation dans une perspective de professionnalisation entre acteurs praticiens et chercheurs. Formation et profession, 20(3), 41-52.

L'écuyer, R. (1990). Méthodologie de l'analyse développementale de contenu. Méthode GPS et concept de soi. Québec, QC : Presses de l'Université du Québec.

Larose, F. et Lenoir, Y. (1998). La formation continue d'enseignants du primaire à des pratiques interdisciplinaires: résultats de recherches. Revue des sciences de l'éducation, 24(1), 189-228. doi: 10.7202/031967ar

Logan, S. et Medford, E. (2011). Gender differences in the strength of association between motivation, competency beliefs and reading skill. Educational Research, 53(1), 85-94.

Lowe, A. (2002). La pédagogie actualisante ouvre ses portes à l'interdisciplinarité scolaire. Éducation et francophonie, 30(2), 188219. 


\section{$\&$}

\section{REVUE HYBRIDE DE L'ÉDUCATION}

McGeown, S., Goodwin, H., Henderson, N. et Wright, P. (2012). Gender differences in reading motivation: does sex or gender identity provide a better account? Journal of Research in Reading, 35(3), 328-336.

Ministère de l'Éducation, du Loisir et du Sport (2009). Progression des apprentissages au primaire. Français, langue d'enseignement. Repéré à http://www1.mels.gouv.qc.ca/progressionPrimaire/francaisEns/pdf/fr aEns SectionCom.pdf.

Mucherah, W. et Yoder, A. (2008). Motivation for reading and middle school students' performance on standardized testing in reading. Reading Psychology, 29, 214-235.

Paillé, P. et Mucchielli, A. (2012). L'analyse qualitative en sciences humaines et sociales ( $3^{e}$ éd.). Paris, France : Armand Colin.

Poupart, J. (1997). L'entretien de type qualitatif : considérations épistémologiques, théoriques et méthodologiques. Dans J. Poupart (dir.), La recherche qualitative. Enjeux épistémologiques et méthodologiques (p. 173-210). Montréal, QC : Gaëtan Morin.

Proulx, J. (2004). L'apprentissage par projet. Québec, QC : PUQ.

Ryan, R. M., et Deci, E. L. (2002). Overview of self-determination theory: An organismic dialectical perspective. Dans E. L. Deci et R. M. Ryan (dir.), Handbook of self-determination research (p. 3-33). Rochester, NY : The University of Rochester Press.

Ryan, R. M., et Deci, E. L. (2000). Self-determination theory and the facilitation of intrinsic motivation, social development, and wellbeing. American Psychoiogist, 55(1), 68-78.

Senn, N. (2012). Effective approaches to motivate and engage reluctant boys in literacy. The Reading Teacher, 66(3), 211-220. 


\section{REVUE HYBRIDE DE L'ÉDUCATION}

Serafini, F. (2013). Supporting boys as readers. The Reading Teacher, 67(1), 40-42. doi:10.1002/TRTR.1187

Sharp, C., Blackmore, J., Kendall, L., Greene, K., Keys, W., Macauley, A., ... Yeshanew, T. (2003). Playing for Success, An Evaluation of the Fourth Year. Nottingham, Royaume-Uni : National Foundation for Educational Research.

Simard, C., Dufays, J.-L., Dolz, J. et Garcia-Debanc, C. (2010). Didactique du français langue première. Bruxelles, Belgique: De Boeck.

Stoloff, S. et Beaudoin, S. (2012). Regards croisés sur la collaboration des acteurs en recherche-action: enjeux et défis. Recherches qualitatives - Les enjeux méthodologiques des recherches participatives, 13, 20-34.

Viau, R. (2009). La motivation à apprendre en milieu scolaire. SaintLaurent, QC : ERPI. 